\title{
Estimating the Number of Men Who Have Sex with Men by Race/Ethnicity at the County Level in Texas
}

\author{
Jesse Campagna, Jonathon Poe, Ann Robbins, \\ and Emily Rowlinson
}

\begin{abstract}
This analysis presents a method for estimating the population of men who have sex with men (MSM) at the county and metropolitan area level in Texas. Surveillance data consistently demonstrate that MSM experience a high burden of HIV and other sexually transmitted infections (STIs). Numerous studies have shown that MSM are also vulnerable to many other health concerns such as suicide, substance abuse, domestic violence and assault, homelessness, and mental illness. However, compilation of rates of HIV, STIs, and other health issues is dependent on estimation of population denominators. In the absence of systematic, consistent, and direct assessment of sexual orientation and gender identity in national surveys, it is difficult to estimate the size of at-risk populations. Previous estimates at the national and state level have been calculated using varied methodologies. However, to date, statewide estimates at the county level have only been produced for the state of Florida. Countylevel and metropolitan area estimates of MSM population were produced using three modified models developed by Lieb et al. These models used data on population and same-sex households from the US Census, along with estimates of sexual behavior from the National Survey on Family Growth. These models produce an estimate of 599,683 MSM in Texas (6.4\% of the adult male population). Metropolitan areas with the highest percentage of MSM population include Dallas and Austin (10.3 and $9.8 \%$, respectively). County-level estimates of MSM population range from 1.0 to $12.9 \%$. These local estimates are critical to targeting vulnerable populations and effective allocation of resources for prevention and treatment programs.
\end{abstract}

KEYWORDS MSM, Texas, Men who have sex with men, HIV surveillance, Population estimates

\section{INTRODUCTION}

Gay men and other men who have sex with men (MSM) continue to experience the highest burden of HIV compared to other groups. MSM are the only group in which the incidence of new HIV diagnoses has been steadily increasing since the epidemic plateaued in the 1990s. ${ }^{1}$ Surveillance data suggests that MSM are becoming the highest-risk group for syphilis infection. ${ }^{2}$ Recent studies have also reported increasing numbers of gonorrhea among MSM, ${ }^{3}$ and suggest that both gonorrhea and chlamydia are severely underestimated in MSM.,5

In addition to sexually transmitted infections (STIs), MSM are vulnerable to a number of other health concerns. MSM and gay men are at greater risk for

Campagna, Poe, Robbins, and Rowlinson are with the Texas Department of State Health Services, PO Box 149347Austin, TX 78756, USA.

Correspondence: Emily Rowlinson, Texas Department of State Health Services, PO Box 149347Austin, TX 78756, USA. (E-mail: emily.rowlinson@dshs.state.tx.us) 
behavioral health problems, including suicide attempts. ${ }^{6-8}$ Gay men have higher rates of clinical eating disorders compared to heterosexual men., ${ }^{9,10}$ Gay men and MSM, lesbians, and transgender individuals are more likely to use alcohol and drugs and have higher rates of substance abuse compared to the general population, ${ }^{6,7,11-15}$ and MSM are more likely to smoke cigarettes compared to heterosexuals. ${ }^{11,15,16}$ MSM and other sexual minorities are more likely to report experiences of violence and victimization, ${ }^{8,17}$ and gay, lesbian, bisexual, and transgender youth are at greater risk for homelessness than their heterosexual peers. ${ }^{18-21}$

In light of the health disparities described in these studies, development of health policy focusing on MSM, sexual minorities, and transgendered persons should be an important priority for public health. Identifying the scope and impact of these issues in MSM is dependent on the calculation of rates of disease and risk factors among this population. However, the primary barrier to compilation of rates and other metrics is the lack of population denominator data. This stems from a lack of systematically collected health data on sexual orientation and gender identity. The US Census, which provides the most comprehensive population-level data at both the national and local levels, does not include questions that directly ask sexual orientation. Probability sample surveys that attempt to measure sexual orientation are subject to under reporting and nonresponse distortion, leading to an underestimation of the true population. Many efforts to estimate MSM population size have not been conducted with sufficient frequency and rigor because of the difficulty, high cost, and stigma associated with reaching this population. ${ }^{22}$

Lieb et al. developed a set of three models to determine the proportion and number of adult MSM at the state level in each of 17 southern states. ${ }^{7}$ However, states are not homogenous in population demographics, and statewide estimates may not be applicable for each county or metropolitan area. Producing estimates of MSM population at the local level would facilitate assessment of community vulnerability, efficient allocation of resources for prevention and treatment, and more focused efforts to conduct disease surveillance and disrupt transmission. These measures will allow a far better understanding of the scope of the epidemic and vulnerability among MSM and specific race/ethnicity groups within the MSM community. We estimated the MSM population in Texas at the county and metropolitan area level by applying a modified version of Lieb et al.'s models, using 3-year population estimates from the US Census.

\section{METHODS}

Lieb et al.'s models were applied to all Texas counties with published US Census data on the percentage of households with same-sex male unmarried partners. The models were also applied to the major metropolitan areas most severely affected by the HIV/AIDS epidemic. Metropolitan areas were defined as the counties comprising each city designated as a Transitional Grant Area (TGA) or Eligible Metropolitan Area (EMA) by Ryan White programs for purposes of funding allocation. In depth discussion of the methodology behind Lieb et al.'s models can be found in the sourced paper. ${ }^{23}$

County-level population data derived from the 2010 US Census were obtained from the US Center for Health Statistics. ${ }^{24}$ Estimates of the number of same-sex unmarried partners (SSMP) in Texas households were obtained from the 2010 US 
Census American Community Survey. ${ }^{25}$ Although the ACS is administered annually, geographic areas with smaller populations combine 3 or 5 years of data in order to produce statistically valid estimates. One-year census estimates were available for very few Texas counties; 3-year census estimates were available for 74 of Texas' 254 counties, while 5-year census estimates were available for nearly all counties.

Although 3-year estimates were only available for 74 of the 254 counties, these 74 counties account for $89 \%$ of Texas' population and all of its metropolitan areas. Texas' population increased $7 \%$ in the 5-year period of 2008-2012. During the same time period, the population of Texas metropolitan areas increased from $8.2 \%$ in Dallas to $12.3 \%$ in Austin. ${ }^{24}$ Using 3-year census estimates provided more timely data than the 5-year estimates while still accounting for the vast majority of Texas' population.

\section{Model A}

Model A assumes the proportion of men who are MSM is consistent in all rural (1 $\%$ ) suburban (4\%), and urban (9\%) urban settings. ${ }^{26}$ We applied these proportions to state and county-level estimates of the male population obtained from the 2000 US Census.

$$
\begin{aligned}
& \text { \% MSM county } \\
& =\left(\text { rural male population county }{ }_{i} \times 0.01\right) \\
& +\left(\text { suburban male population county }{ }_{i} \times 0.04\right) \\
& +\left(\text { urban male population } \text { county }_{i} \times 0.09\right)
\end{aligned}
$$

\section{Model B}

Lieb's Model B assumes the "relative concentration of same-sex male unmarried partners (SSMP) in a state's households is an indicator of the concentration of MSM in the state." 22 Similarly, we assumed that the relative concentration of SSMP in a county's households would therefore be an indicator of the concentration of MSM in that county.

This model first calculates a ratio of a Texas county's proportion of SSMP in Texan households to the county's proportion of households in Texas, producing an MSM index.

MSM index county $_{i}$

$$
=\left(\# \text { SSMP county }{ }_{i} / \# \text { SSMP Texas }\right) /\left(\# \text { households county } y_{i} / \# \text { households Texas }\right)
$$

Lieb's model B multiplies the national estimate of the percentage of men who are MSM obtained from the US National Survey on Family Growth (NSFG) $(6 \%)^{26}$ by state-level MSM Indices to obtain the MSM population for each state. However, NSFG did not produce estimates of MSM at the Texas state and county levels; therefore, the county-level proportions of MSM derived in model A were applied to each corresponding county's MSM index.

Percent MSM county ${ }_{i}=$ MSM index county $_{i} \times \%$ MSM Model A county ${ }_{i}$

County-level estimates of the proportion of adult males ( $\geq 18$ years old) who are MSM were then calculated by averaging the results of Models A and B. 
Average $\%$ MSM county $_{i}=\left(\%\right.$ MSM model A county $_{i}+\%$ MSM model B county $\left._{i}\right) / 2$

Final county-level estimates of the number of adult MSM were calculated by multiplying this county-level proportion of MSM by the county-level adult $(\geq 18$ years) male population estimate.

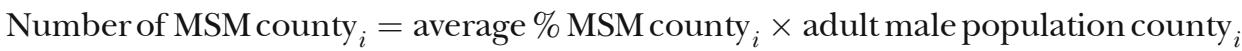

\section{Model C}

Model C calculates race-/ethnicity-specific estimates of MSM at the county level. Lieb calculated ratios of the percentage MSM for blacks, Hispanics, and other racial groups using whites as the reference group. ${ }^{26}$ We assumed that these ratios would apply to all counties within Texas. Each race-specific ratio was multiplied by the estimated percent MSM for each county obtained in models A and B (which includes males of all races) and the county's total male population for that race.

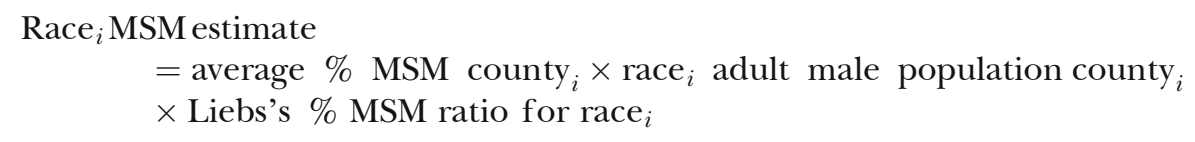

These race-/ethnicity-stratified adult MSM estimates were summed to produce final county-level estimates.

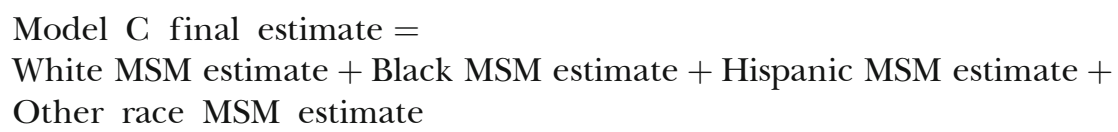

The number of MSM living with HIV was compared to the estimates of MSM population in Texas counties by race to assess the validity of the methodology used to produce these estimates. The number of MSM living with HIV was comprised of the cumulative count of individuals living with HIV in Texas who are not known to be deceased with an identified or imputed risk factor of male-male sexual contact. ${ }^{27}$ Data on the number of MSM living with HIV was obtained from Texas' enhanced HIV/AIDS reporting system (eHARS).

\section{RESULTS}

\section{Estimates}

We estimate that in 2012, Texas had an adult MSM population of 599,683 or $6.4 \%$ of males $\geq 18$ years of age. The racial breakdown of the MSM population includes 315,000 (53 \%) Whites, 56,000 (9 \%) Blacks, 213,000 (36\%) Hispanic/Latinos, and 16,000 (3\%) men of other races. The estimates show considerable variability in county-specific racial/ethnic percentages of MSM (Tables 1 and 2).

The largest populations of MSM populations reside in metropolitan areas with the highest concentrations in the Dallas and Austin metropolitan areas (10.3 and $9.8 \%$ respectively). In Dallas and Travis counties, the two central counties in these 


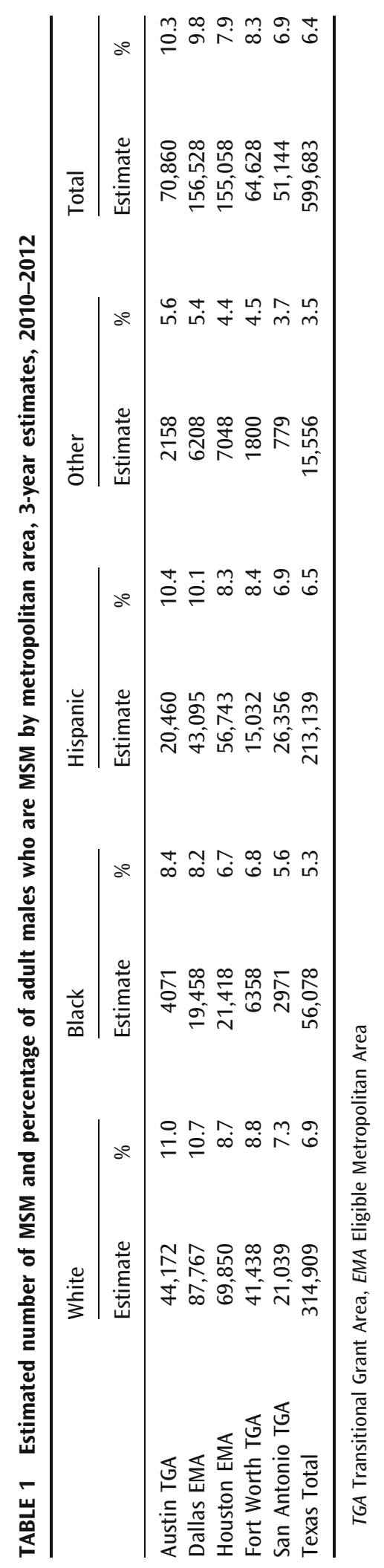




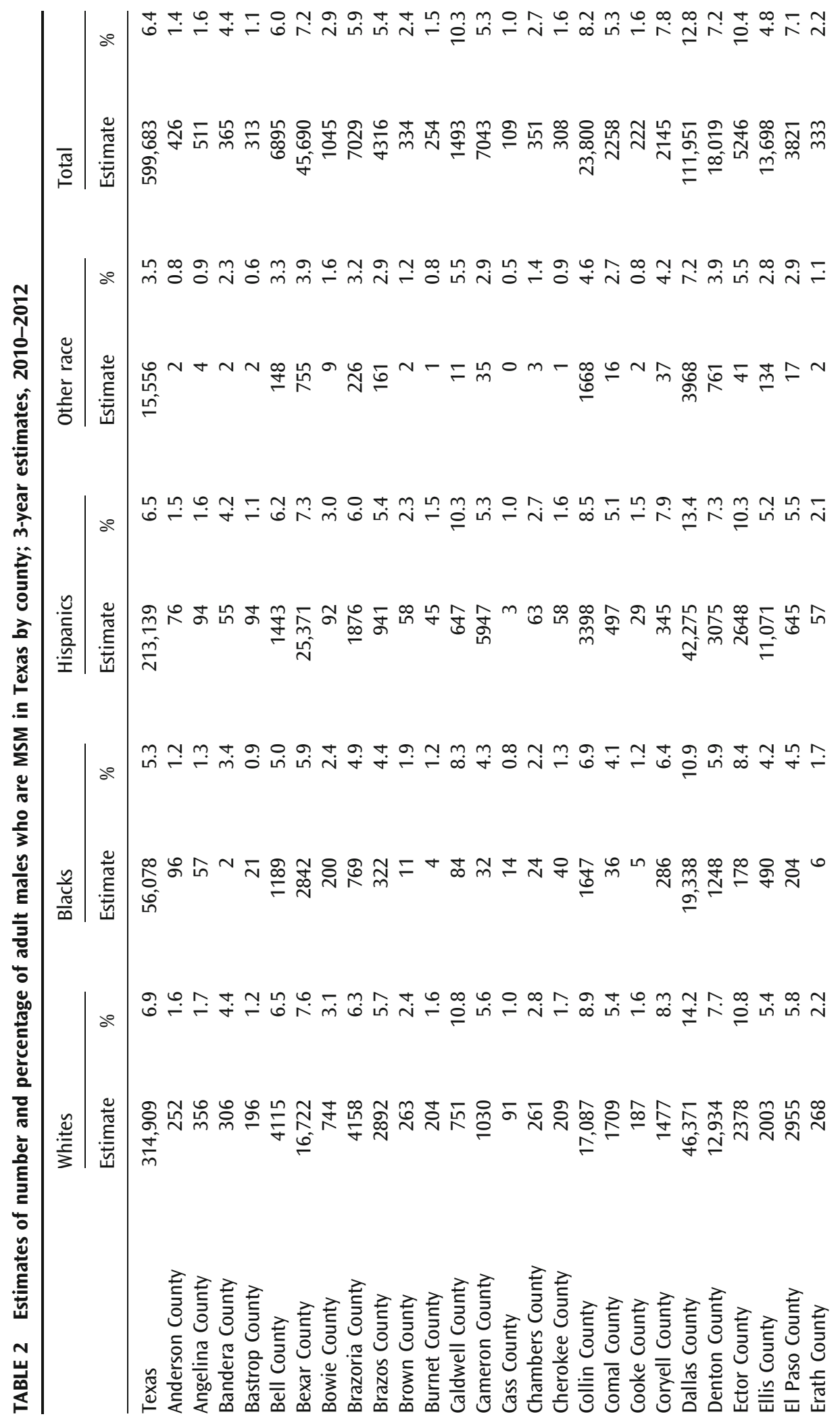




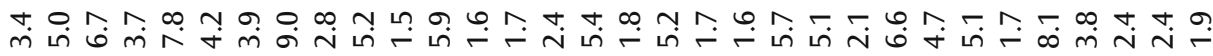

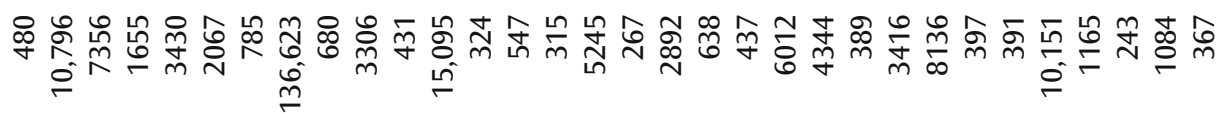

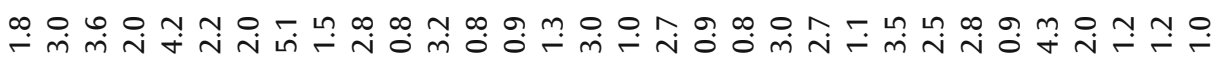

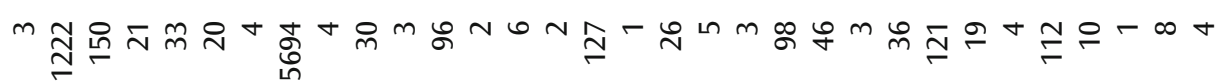

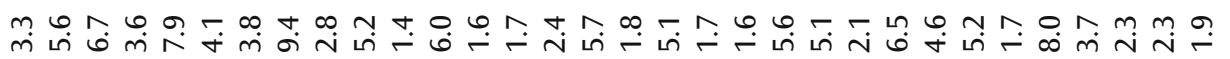

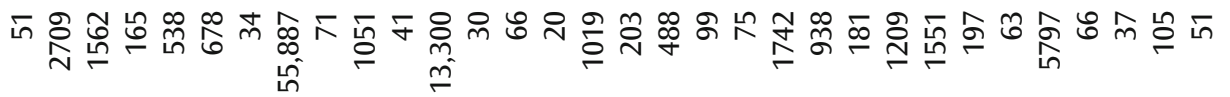

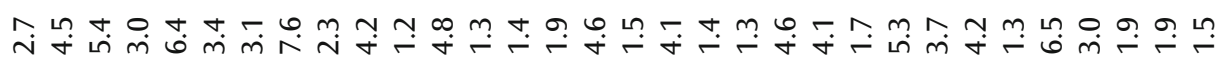

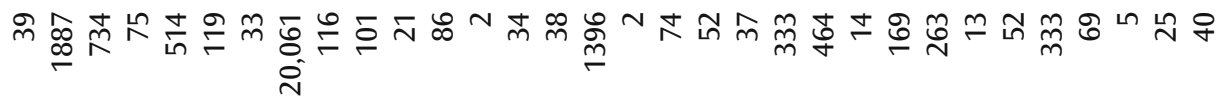

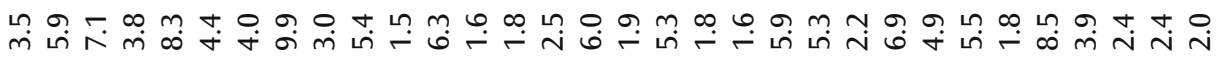

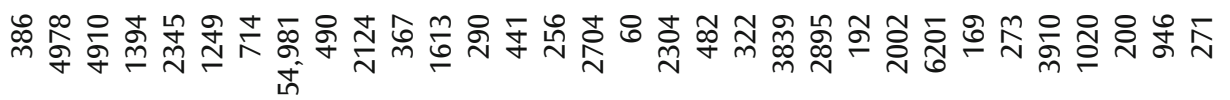

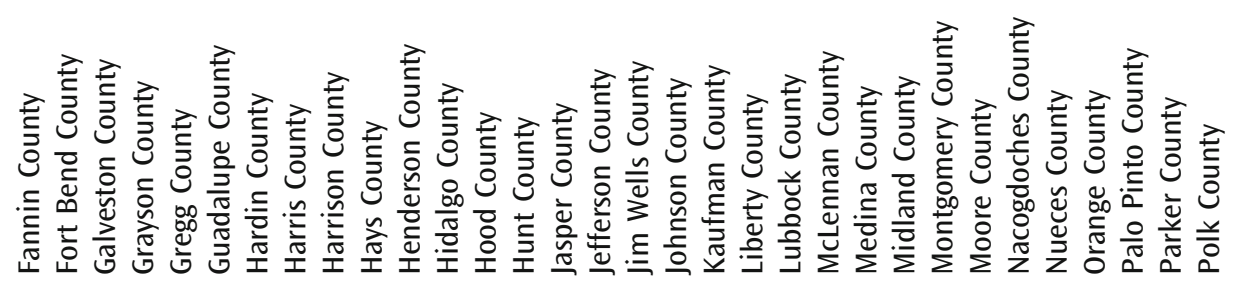




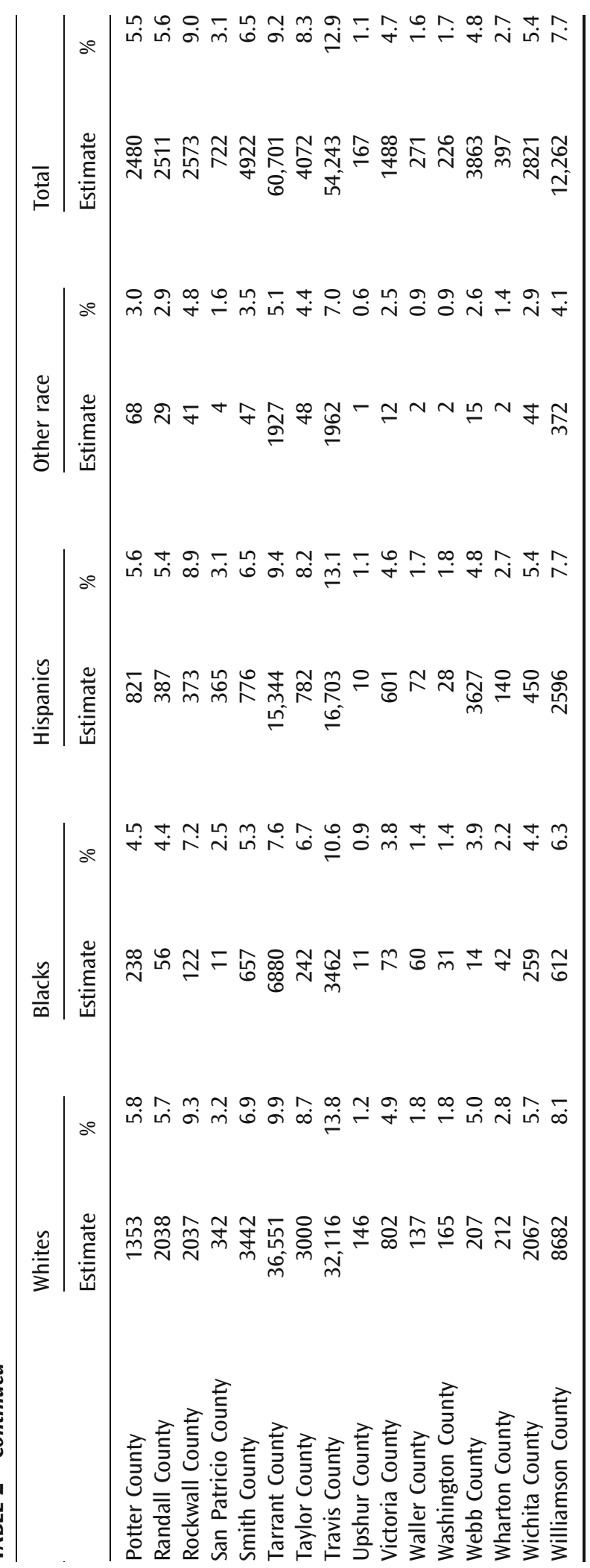


metropolitan areas, MSM comprise 12.8 and $12.9 \%$ of all male residents, respectively (Table 2). Only two counties, Dallas and Harris (where Houston is located), had adult MSM populations exceeding 100,000 (111,951 for Dallas and 136,623 for Harris).

\section{MSM Living with HIV}

The number of MSM living with HIV in Texas in 2012 was estimated to be 41,774, which corresponds to approximately $6.6 \%$ of our estimated MSM population. This is similar to a previous published estimate of the percentage of Texan MSM living with HIV $(7.0 \%)^{28}$ and several other estimates in other MSM populations using different methodologies. ${ }^{29-31}$ In all individual counties and metropolitan areas (Table 3) for which MSM population estimates were calculated, the number of MSM living with HIV did not exceed the MSM population, both in total and stratified by race/ethnic group.

\section{DISCUSSION}

While individual studies point to significant health disparities for MSM and other sexual minorities, a comprehensive understanding of the scope of inequities faced by these populations cannot be created solely through special studies of individual health conditions. The development of evidence-based health policy is traditionally guided by population based health and demographic surveys which collect crucial data on social determinants of health, including social, economic, and environmental factors. However, in the absence of systematic, consistent, and direct assessment of sexual orientation and gender identity in such surveys, it is impossible to accurately assess social determinants of health in sexual minorities and transgender persons. $^{32,33}$

In recent years, numerous medical and governmental institutions have called for routine collection of sexual orientation data in various national surveys. In its 2011 report The Health of Lesbian, Gay, Bisexual, and Transgender People: Building a Foundation for Better Understanding, the Institute of Medicine (IOM) recommended collection of gender identity and sexual orientation data on all federally supported surveys. ${ }^{34}$ Section 4302 of the Affordable Care Act contains provisions to strengthen assessment of health status and inequities, including a plan to integrate sexual orientation and gender identity variables into all Health and Human Services national surveys. In 2013, the National Health Interview Survey (NHIS) included questions about sexual orientation along with topics historically included on this

TABLE 3 Number of MSM living with HIV by metropolitan area, 2012

\begin{tabular}{lrrrrr}
\hline & White & Black & Hispanic & Other & Total \\
\hline Austin TGA & 1767 & 408 & 1036 & 30 & 3325 \\
Dallas EMA & 5060 & 3502 & 2785 & 124 & 11,857 \\
Houston EMA & 3851 & 4237 & 3675 & 173 & 12,199 \\
Fort Worth TGA & 1076 & 740 & 538 & 40 & 2498 \\
San Antonio TGA & 888 & 380 & 2160 & 27 & 3568 \\
Texas Total & 15,224 & 10,986 & 13,944 & 1195 & 41,774 \\
\hline
\end{tabular}


household survey (e.g., health insurance, vaccination, obesity). Efforts to add sexual orientation and gender identity are expected to continue, as outlined in the developmental goals in the Healthy People 2020 for the new topic area of lesbian, gay, bisexual and transgender health.

For individual patient care, there are no standards or Meaningful Use Guidelines for collecting information on sexual orientation or gender identity, although the documentation of such information in electronic health records is recommended in the 2011 IOM report and Healthy People 2020 goals referenced above and the Joint Commission's 2010 report, Advancing Effective Communication, Cultural Competence, and Patient- and Family-Centered Care: A Roadmap for Hospitals. Systematic and standard inclusion of sexual orientation and gender identity information in clinical settings would provide data that could be used to improve care delivery and inform efforts to reduce health disparities in these groups outside of the traditional domains of (STIs) and HIV.

Until national surveys and medical records guidelines are revised to routinely collect such information, methodologies such as those used in this paper are the best source of population estimation available. The primary application for estimates produced by these methods is their use as denominators for estimating the incidence and prevalence rates of HIV/ STIs and other diseases and risk factors in MSM populations. Estimation of these and other metrics will assist in more effective allocation of funds and services and targeted interventions.

We have demonstrated that estimates of MSM can be produced and updated by state and local health departments and community organizations using publicly accessible population data from the US Census. Model A relies upon decennial US Census data last collected in 2010, but model B can be revised annually with updated ACS data. Producing timely estimates at the county and metropolitan area will depend on the local population size, as annual estimates of SSMP are only published for highly populated areas. This may limit the ability of rural states to produce accurate estimates of the MSM populations residing outside of urban areas.

Our statewide estimate of $6.4 \%$ MSM population differs only slightly from that of Lieb et al.'s published estimate of $6.3 \%$. However, our estimate is much lower than the recently published NHIS' national estimate of $1.8 \%{ }^{35}$ Given the imprecision of methods which rely on proxies for sexual orientation, it is difficult to know whether any these estimates are accurate. Verification of these estimates would be best accomplished through the development and implementation of data collection tools and survey methodologies, several of which are discussed below.

Surveillance data on the number of MSM living with HIV in Texas did not exceed our estimates of the MSM population in Texas, even when stratified by county and race. This would indicate that we did not overestimate the number of MSM living in these counties. This population data will allow for calculation of locally relevant HIV prevalence and new diagnosis rates. Texas also routinely collects sexual orientation information from patients with syphilis infection through public health follow-up programs. This surveillance data can be used in conjunction with the MSM population estimates to calculate syphilis incidence rates among MSM. Currently, sexual orientation is not routinely collected and reported for gonorrhea and chlamydia cases; however, future surveillance 
activities, including rectal screening for STIs and public health follow-up for gonorrhea infections in MSM, may allow for calculation of rates of infection in MSM.

\section{LIMITATIONS}

We faced several limitations when producing our estimates of MSM population. The two major barriers to determining the proportion of MSM in a region are a lack of sexual orientation data and the compounded margin of error created when generating estimates based upon other estimates.

There is no major reliable source or survey that specifically explores the sexual orientation or behavior of the population in the Texas. We relied on data from the US Census, specifically the percentage of household with same-sex unmarried partners, to serve as a proxy for the concentration of MSM in a particular county. The US Census and American Community Survey (ACS) derives statistics on same-sex couple households using two census questions, relationship to householder and sex of each person in the household. The US Census specifies, "The wrong box may have been checked for the sex of a small percentage of oppositesex spouses and unmarried partners." ${ }^{36}$ More importantly, stigma may have led to males inaccurately reporting their relationship to the head of household. The first scenario could result in over or underestimation of the percentage of households with same-sex unmarried partners, while the latter would have resulted in an underestimation.

Three-year census estimates of households with same-sex unmarried partners were only available for 74 of 254 counties. The counties for which 3 -year census estimates were not available are mainly rural and only account for $11 \%$ of the state's population. Our analysis shows that urban and highly populated counties tend to have higher percentage MSM populations compared to rural counties. The lack of 3-year census estimates from 180 mostly rural counties likely resulted in an underestimation of state MSM population in models using these census estimates. Additionally, data on the proportion of households located in urban, suburban, and rural areas are only updated during the decennial U.S. Census. Texas has experience unprecedented population growth in the past decade, especially in urban areas, and the lack of recent data may impact the accuracy of estimates produced by Model A.

We could find no published state-level estimates for several variables used in Lieb's models. For both the proportion of males who are MSM in rural, suburban, and urban areas and by race/ethnic groups, we relied on national estimates from the most recent NSFG. The true value of these estimates may be different in Texas. The methodology used in model A relied solely on the rural/urban/suburban composition of the county. In majority rural counties, this methodology likely produced an underestimate of the total MSM population. Similarly, model A likely produced overestimates in heavily urban counties. However, with the aforementioned lack of state-level data on sexual orientation, we have no way to determine the degree to which the national estimates may or may not be applicable to Texas. 


\section{CONCLUSION}

Creating estimates of the MSM population in Texas counties and metropolitan areas provides Texas State and local health workers with the essential denominators used to calculate both prevalence and incidence estimates. Accurate assessment of disease burden in MSM is an important tool in the mission to better serve this population. These estimates may be used to guide future outreach, efficiently allocate resources, and better target HIV/AIDS and STI surveillance efforts. While estimation methods such those outlined in this paper are needed to provide context for STI and HIV, they are not a satisfactory long-term substitute for the standardized and systematic inclusion of information on sexual orientation that would allow development of public health policies to improve the whole health of sexual minorities and transgendered persons.

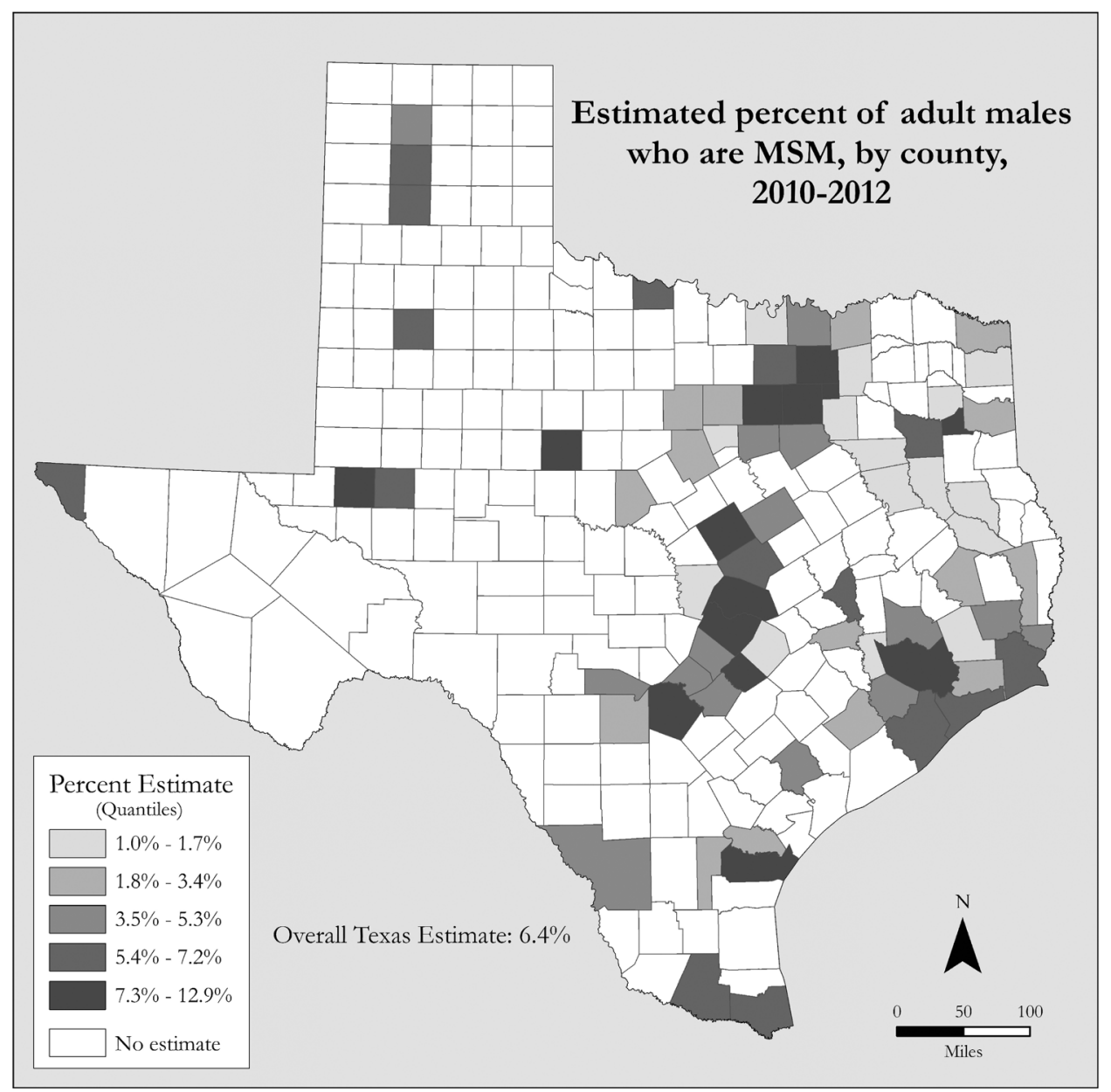




\section{REFERENCES}

1. Prejean J, Song R, Hernandez A, et al. Estimated HIV incidence in the United States, 2006-2009. PLoS One. 2011;6(8):e17502. doi:10.1371/journal.pone.0017502.

2. Centers for Disease Control and Prevention. Notes from the field: repeat syphilis infection and HIV coinfection among men who have sex with men-Baltimore, Maryland, 20102011. MMWR. 2013;62:649-50.

3. Center for Disease Control. Sexually transmitted disease surveillance 2007 Supplement, Gonococcal Isolate Surveillance Project (GISP) Annual Report 2007.; 2009.

4. Kent CK, Chaw JK, Wong W, et al. Prevalence of rectal, urethral, and pharyngeal chlamydia and gonorrhea detected in 2 clinical settings among men who have sex with men: San Francisco, California, 2003. Clin Infect Dis. 2005;41:67-74. doi:10.1086/ 430704.

5. Gunn RA, O’Brien CJ, Lee MA, Gilchick RA. Gonorrhea screening among men who have sex with men: value of multiple anatomic site testing, San Diego, California, 1997-2003. Sex Transm Dis. 2008;35:845-8. doi:10.1097/OLQ.0b013e318177ec70.

6. Ibañez GE, Purcell DW, Stall R, Parsons JT, Gómez CA. Sexual risk, substance use, and psychological distress in HIV-positive gay and bisexual men who also inject drugs. AIDS. 2005;19(Suppl 1):S49-55. doi:10.1097/01.aids.0000167351.00503.92.

7. Herek GM, Garnets LD. Sexual orientation and mental health. Annu Rev Clin Psychol. 2007;3:353-75. doi:10.1146/annurev.clinpsy.3.022806.091510.

8. Roberts AL, Austin SB, Corliss HL, Vandermorris AK, Koenen KC. Pervasive trauma exposure among US sexual orientation minority adults and risk of posttraumatic stress disorder. Am J Public Health. 2010;100:2433-41. doi:10.2105/AJPH.2009.168971.

9. French SA, Story M, Remafedi G, Resnick MD, Blum RW. Sexual orientation and prevalence of body dissatisfaction and eating disordered behaviors: a population-based study of adolescents. Int J Eat Disord. 1996;19:119-26. doi:10.1002/(SICI)1098108X(199603)19:2<119::AID-EAT2>3.0.CO;2-Q.

10. Siever MD. Sexual orientation and gender as factors in socioculturally acquired vulnerability to body dissatisfaction and eating disorders. J Consult Clin Psychol. 1994;62:252-60. doi:10.1037/0022-006X.62.2.252.

11. Ostrow DG, Stall R. Alcohol, tobacco, and drug use among gay and bisexual men. In: Woliitski RJ, Stall R, Valdiserri RO, eds. Unequal opportunity: health disparities affecting gay and bisexual men in the United States. New York, NY: Oxford University Press; 2008:121-58.

12. Hughes TL. Alcohol use and alcohol-related problems among lesbians and gay men. Annu Rev Nurs Res. 2005;23:283-325.

13. Lyons T, Chandra G, Goldstein J. Stimulant use and HIV risk behavior: the influence of peer support group participation. AIDS Educ Prev. 2006;18:461-73. doi:10.1521/ aeap.2006.18.5.461.

14. Mansergh G, Colfax GN, Marks G, Rader M, Guzman R, Buchbinder S. The Circuit Party Men's Health Survey: findings and implications for gay and bisexual men. Am J Public Health. 2001;91:953-8.

15. Skinner WF. The prevalence and demographic predictors of illicit and licit drug use among lesbians and gay men. Am J Public Health. 1994;84:1307-10. doi:10.2105/ AJPH.84.8.1307.

16. Lee JGL, Griffin GK, Melvin CL. Tobacco use among sexual minorities in the USA, 1987 to May 2007: a systematic review. Tob Control. 2009;18:275-82. doi:10.1136/ tc.2008.028241.

17. Kosciw J, Greytak E, Bartkiewicz M. The 2007 National School Climate Survey: the experiences of lesbian, gay, bisexual and transgender youth in our Nation's Schools.; 2012:1-174. doi:ISBN 978-193409205-7. 
18. Coker TR, Austin SB, Schuster MA. The health and health care of lesbian, gay, and bisexual adolescents. Annu Rev Public Health. 2010;31:457-77. doi:10.1146/ annurev.publhealth.012809.103636.

19. Kruks G. Gay and lesbian homeless/street youth: special issues and concerns. J Adolesc Health. 1991;12:515-8. doi:10.1016/0197-0070(91)90080-6.

20. Van Leeuwen JM, Boyle S, Salomonsen-Sautel S, et al. Lesbian, gay, and bisexual homeless youth: an eight-city public health perspective. Child Welf. 2006;85:151-70.

21. Conron KJ, Mimiaga MJ, Landers SJ. A population-based study of sexual orientation identity and gender differences in adult health. Am J Public Health. 2010;100:1953-60.

22. Lieb S, Thompson DR, Misra S, et al. Estimating populations of men who have sex with men in the Southern United States. J Urban Heal. 2009;86(6):887-901. doi:10.1007/ s11524-009-9401-4.

23. Lieb S, Thompson D, Al E. Estimating populations of men who have sex with men in the Southern United States. J Urban Heal Bull N Y Acad Med. 2009;Published.

24. National Center for Health Statistics. Vintage 2013 postcensal estimates of the resident population of the United States (April 12010 , July 1, 2010-July 1, 2013), by year, county, single-year of age $(0,1,2, . ., 85$ years and over), bridged race, Hispanic origin, and sex. Prepared under a col. Available at: http://www.cdc.gov/nchs/nvss/bridged_race/htm as of June 26, 2014, following release by the U.S. Census Bureau of the unabridged Vintage 2013 postcensal estimates by 5-year age group on June 26, 2014. Accessed 8 July 2014.

25. United States Census Bureau/American FactFinder. "B11009: unmarried partner households by sex of partner.” 2010-2012 American Community Survey. 2013. Available at: http://factfinder2.census.gov. Accessed 30 Apr 2014.

26. Mosher WD, Chandra A, Jones J. Sexual behavior and selected health measures: men and women 15-44 years of age, United States. Adv Data. 2002;2005:1-55.

27. Harrison KM, Kajese T, Hall HI, Song R. Risk factor redistribution of the National HIV/ AIDS Surveillance Data: an alternative approach. Public Health Rep. 2008;123:618-27.

28. Lieb S, Prejean J, Thompson DR, et al. HIV prevalence rates among men who have sex with men in the Southern United States: population-based estimates by race/ethnicity. AIDS Behav. 2011;15:596-606. doi:10.1007/s10461-010-9820-y.

29. Manning SE, Thorpe LE, Ramaswamy C, et al. Estimation of HIV prevalence, risk factors, and testing frequency among sexually active men who have sex with men, aged 18-64 yearsNew York City, 2002. J Urban Health. 2007;84:212-25. doi:10.1007/s11524-006-9135-5.

30. Valleroy LA, MacKellar DA, Karon JM, et al. HIV prevalence and associated risks in young men who have sex with men. Young Men's Survey Study Group. JAMA. 2000;284:198-204. doi:10.1001/jama.284.2.198.

31. Xu F, Sternberg MR, Markowitz L. Men who have sex with men in the United States: demographic and behavioral characteristics and prevalence of HIV and HSV-2 infection: results from National Health and Nutrition Examination Survey 2001-2006. Sex Transm Dis. 2010;37(6):399-405.

32. Dean HD, Fenton KA. Addressing social determinants of health in the prevention and control of HIVIAIDS, viral hepatitis, sexually transmitted infections, and tuberculosis.; 2010:Suppl 4: 1-5. Available at: http://www.cdc.gov/nchhstp/. Accessed 17 Mar 2014.

33. Marmot M, Friel S, Bell R, Houweling TAJ, Taylor S. Closing the gap in a generation: health equity through action on the social determinants of health. Lancet. 2008;372:1661-9. doi:10.1016/S0140-6736(08)61690-6.

34. Institute of Medicine. The health of lesbian, gay, bisexual, and transgender people: building a foundation for better understanding. Washington DC: Press TNA; 2011.

35. Ward BW, Dahlhamer JM, Galinsky AM, Joestl SS. Sexual orientation and health among U.S. adults: National Health Interview Survey, 2013. Natl Health Stat Rep. 2014:1-12. Available at: http://www.ncbi.nlm.nih.gov/pubmed/25025690. Accessed 23 May 2014.

36. US Census Press Release. Census bureau releases estimates of same sex married couples. Sept. 27, 2011. Available at http://www.census.gov/2010census/news/releases/operations/ cb11-cn181.html. Accessed 17 Oct 2013. 